\title{
CROP PREDICTION USING DEEP NEURAL NETWORK
}

\section{MANPRIYA $^{1} \&$ DR. VIKAS JINDAL ${ }^{2}$}

${ }^{I}$ ME Scholar, Computer Science and Engineering, Chandigarh University, Mohali (Punjab), India

${ }^{2}$ Professor, Computer Science and Engineering, Chandigarh University, Mohali (Punjab), India

\begin{abstract}
Agriculture played an important role in Indian economy. Technology in agriculture is still very new in India. This paper builds a crop prediction model using deep learning. In most of the previous research works, machine learning was used for crop prediction and few limited crops are included. Our model is built using Deep Neural Network to predict appropriate crop for every district of India. 124 crops are included in our work. Predictions are made on the basis of dataset taken from Indian government agricultural portal. $75 \%$ of dataset used as training dataset and remaining $25 \%$ is used as test dataset. Sigmoid Activation function and SGD optimizer is used in this work. This model gave the accuracy of $99.19 \%$.
\end{abstract}

KEYWORDS: Crop Prediction, Deep Learning, Deep Neural Network

Received: May 20, 2020; Accepted: Jun 10, 2020; Published: Jul 24, 2020; Paper Id.: IJMPERDJUN2020435

\section{INTRODUCTION}

Economy of India generously depends upon the Agricultural sector of the country. Agricultural practices had been an income source for most of the Indians from days of yore which makes the Indian economy generously dependable on agriculture. More than 1.6 million square-kilometres of India's land area are used for cultivation purpose. In recent times, farmers are struggling a lot in making right choices of crops to be sown at appropriate season for better production of crop. Technology in agriculture is still very new in India as most of the agricultural practices are in rural areas. Due to the lack of knowledge regarding crop selection, the farmers suffer loss which leads to increase the number of suicidal cases of farmers [1].

Crop Prediction is of excessive importance to total food production [2]. Hence, there is a need of effective crop prediction technique for better crop production [3]. Various machine learning algorithms are used for predicting crop on the basis of various factors. Some of them are: Random forest, K-nearest neighbour, Support vector machine, Naïve Bayes, etc. Machine Learning is an arena of training that offers system to self-learn and progress from experience. Machine learning is an effective method to provide optimized crop prediction for the farmers [5].

Deep Learning is a subclass of machine learning based on the Artificial Neural Networks. Our proposed work uses Deep Neural Network to train model for the prediction of crops. Predictions are made on the basis of dataset taken from Indian government agricultural portal [17]. Sigmoid Activation function is used for mathematical computation and SGD optimizer is used for updating parameters and weights to reduce loss function in this work. 
The assembly of this paper is as follows: Section II contains Related Work i.e. brief review of previous work. Section III is Methodology which further includes dataset, data pre-processing and prediction. Section IV includes the Experiment Results and lastly, Section V is Conclusion and References.

\section{RELATED WORK}

In [1], considering south Indian states, a Hybrid Crop recommender system was proposed that uses classifier algorithms like Naïve Bayes, J48 and association rules. In this work, recommendations are made based on the climatic and geographical features. In this work, Naïve Bayes gave accuracy of $69.90 \%$ and J48 classifier gave accuracy of $95.93 \%$.

A crop recommender system was proposed in [7], recommending crop and the ratio of fertilizer required according to soil and atmospheric features. Crop and fertilizer sample data is used for recommendation. This model uses classification algorithms like Random Forest and Back Propagation. Back propagation gave higher error rate than Random forest.

An ensembling technique was used in [8] to builda Crop Recommender System. In this work, ensembling technique used base learner like Naïve Bayes, Random Forest and linear SVM. The class labels of each classifier are combined by the use of majority voting. $99.91 \%$ of average accuracy was obtained in this work.

A Crop Recommender System was proposed in [9] that used satellite images to recommended crop on the basis of environmental factors. The researcher proposed a modified CNN which used spatial features as input. The model was trained using back propagation. The error rate of prediction was reduced in this work.

In [10], researcher proposed an expert system for the farmer that helps them to know which crop should be sown at outlined location. This model also provides knowledge about the diseases on plants. To detect the location for gaining information about the weather condition, soil type, crop pattern and groundwater information, etc. GPS system was used. The classifiers used in this model are: Convolutional Neural Network and Recurrent Neural Network.

'AgroConsultant' named recommender system was proposed in [11] which predicts the crop to be sown on the basis of various parameters like season, soil type, geographical location, rainfall and temperature. 21 numbers of crops were included in this work. Neural Network was used in this work which provided the accuracy of $91 \%$.

In [13], the researcher proposed a recommender system that used ensembling technique and make prediction on the basis of soil type. In this work, researchers used 9 crops for prediction. This work used Support Vector Machine (SVM), Multi-Layer Perceptron (ANN), Naïve Bayes, and Random Forest as base learners.

The Researcher used ensembling model to design a recommender system in [14]. The numbers of crops used in this work are 10. In this work, majority voting technique was used to combine class labels of each of CHAID, Random Forest, Naïve Bayes, and KNN classifiers. $88 \%$ of accuracy was achieved by this model.

In [15], a recommender system was proposed which suggests the crops and fertilizers for growing productivity on the basis of the condition of the land. This model makes prediction for 9 minor crops. ANN was used for modelling and prediction. $90 \%$ of accuracy was attained by this work. 


\section{METHODOLOGY}

In this work, a crop prediction model is designed using Deep Neural Network. The dataset used in this work is taken from Indian government agricultural portal [17]. The framework of proposed work is illustrated in Figure 1.

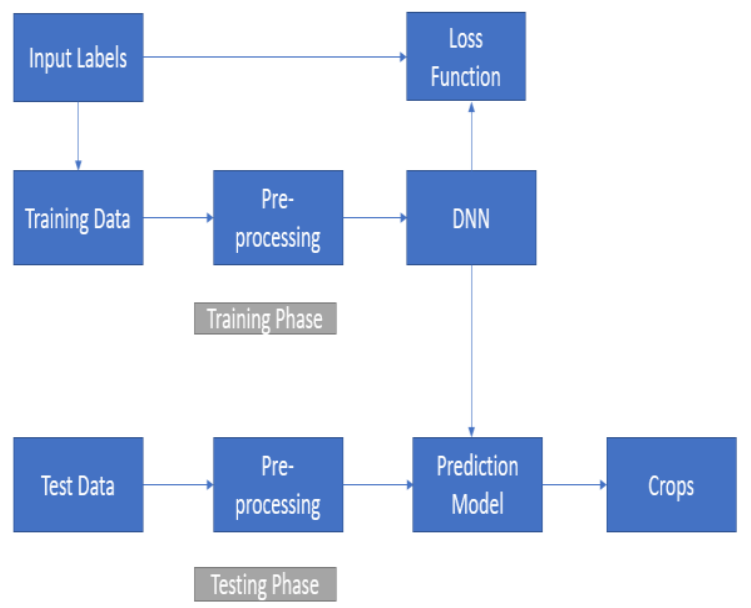

Figure 1: Basic Framework of Proposed Work.

\section{Dataset}

The dataset used in this work is taken from Indian government agricultural portal [17]. This dataset includes parameters like state name, district, crop, season, area and production. This data carries the crop production data of various districts of each state of India from 1997 to 2015 . This dataset includes 124 crops. Table 1 illustrates the dataset statistics.

\section{Table 1: Dataset Statistics}

\begin{tabular}{|l|c|}
\hline Number of Crops & 124 \\
\hline Number of States & 28 \\
\hline Number of UTs & 7 \\
\hline Number of Seasons & 5 \\
\hline
\end{tabular}

The sample of the dataset taken from Indian government agricultural portal is illustrated in Figure 2.

\begin{tabular}{|c|c|c|c|c|c|c|}
\hline 1 & State_Name & | District_Name & p_Year Season & Crop & Area & Production \\
\hline 2 & Andaman and Nicobar Islands & NICOBARS & 2000 Kharif & Arecanut & 1254 & 2000 \\
\hline 3 & Andaman and Nicobar Islands & NICOBARS & 2000 Kharif & Other Kharif, & 2 & \\
\hline 4 & Andaman and Nicobar Islands & NICOBARS & 2000 Kharif & Rice & 102 & 321 \\
\hline 5 & Andaman and Nicobar Islands & NICOBARS & 2000 Whole Year & Banana & 176 & 641 \\
\hline 6 & Andaman and Nicobar Islands & NICOBARS & 2000 Whole Year & Cashewnut & 720 & 165 \\
\hline 7 & Andaman and Nicobar Islands & NICOBARS & 2000 Whole Year & Coconut & 18168 & 65100000 \\
\hline 8 & Andaman and Nicobar Islands & NICOBARS & 2000 Whole Year & Dry ginger & 36 & 100 \\
\hline 9 & Andaman and Nicobar Islands & NICOBARS & 2000 Whole Year & Sugarcane & 1 & \\
\hline 10 & Andaman and Nicobar Islands & NICOBARS & 2000 Whole Year & Sweet potatc & 5 & 15 \\
\hline 11 & Andaman and Nicobar Islands & NICOBARS & 2000 Whole Year & Tapioca & 40 & 169 \\
\hline 12 & Andaman and Nicobar Islands & NICOBARS & 2001 Kharif & Arecanut & 1254 & 2061 \\
\hline 13 & Andaman and Nicobar Islands & NICOBARS & 2001 Kharif & Other Khariff & 2 & \\
\hline 14 & Andaman and Nicobar Islands & NICOBARS & 2001 Kharif & Rice & 83 & 300 \\
\hline 15 & Andaman and Nicobar Islands & NICOBARS & 2001 Whole Year & Cashewnut & 719 & 192 \\
\hline 16 & Andaman and Nicobar Islands & NICOBARS & 2001 Whole Year & Coconut & 18190 & 64430000 \\
\hline 17 & Andaman and Nicobar Islands & NICOBARS & 2001 Whole Year & Dry ginger & 46 & 100 \\
\hline 18 & Andaman and Nicobar Islands & NICOBARS & 2001 Whole Year & Sugarcane & 1 & \\
\hline 19 & Andaman and Nicobar Islands & NICOBARS & 2001 Whole Year & Sweet potatc & 11 & 33 \\
\hline 20 & Andaman and Nicobar Islands & NICOBARS & 2002 Kharif & Rice & 189.2 & 510.84 \\
\hline 21 & Andaman and Nicobar Islands & NICOBARS & 2002 Whole Year & Arecanut & 1258 & 2083 \\
\hline 22 & Andaman and Nicobar Islands & NICOBARS & 2002 Whole Year & Banana & 213 & 12/8 \\
\hline 23 & Andaman and Nicobar Islands & NICOBARS & 2002 Whole Year & Black pepper & 63 & 13.5 \\
\hline 24 & Andaman and Nicobar Islands & NICOBARS & 2002 Whole Year & Cashewnut & 719 & 208 \\
\hline 25 & Andaman and Nicobar Islands & NICOBARS & 2002 Whole Year & Coconut & 18240 & 490000 \\
\hline 26 & Andaman and Nicobar Islands & NICOBARS & 2002 Whole Year & Dry chillies & 413 & 28.8 \\
\hline 27 & Andaman and Nicobar Isle & NICOBARS & 2002 Whole Year & Dry ginger & 47.3 & 133 \\
\hline 28 & Andaman and Nicobar I & NICOBARS & 2002 Whole Year & Sugarcane & 5 & 40 \\
\hline 29 & Andaman and Nicobar Islands & NICOBARS & 2003 Kharif & Rice & 52 & 90.17 \\
\hline
\end{tabular}

Figure 2: Sample of the Dataset Used. 


\section{Preprocessing}

The obtained dataset is converted into numeric data. The dataset is split into training and test dataset. $75 \%$ of data is for training and the remaining $25 \%$ is used for validation. Label Encoder () was used to transform text data into numeric labels. It is also used for normalization. 2D-matrix of the data is created.

\section{Deep Neural Network}

Deep Neural Network obliges as compact outline to Deep Learning. In DNN, multiple layers of neurons are connected passing signals in single direction. From the training dataset, the model is trained to acquire the correlation among the given input and the output. Corresponding to the given set of input, a set of output is obtained by using feed-forward learning through multiple hidden layers.

We have trained our model using DNN having one input layer, two hidden layers and one output layer. We have used sigmoid as activation function.SGD optimizer is used for updating parameters and weights to reduce loss function in this work.

A Deep Neural Network Architecture is illustrated in Figure 3. Here, we input the State, District and Season to the network and on the basis of the production of crop from 1997 to 2015, crops are predicted.

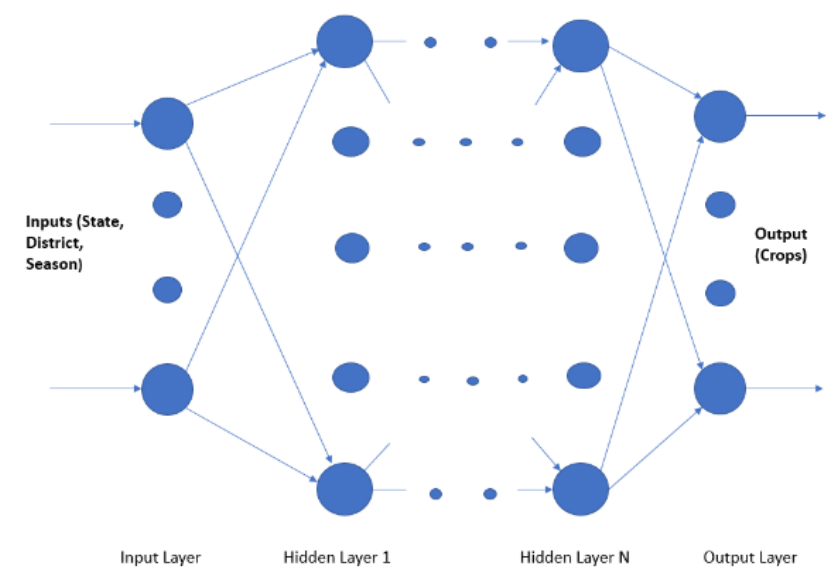

Figure3: Deep Neural Network Architecture.

\section{Prediction}

The crop prediction is made on the basis of the production of crop in different districts from 1997 to 2015 . Finally, the crop prediction is made for various season and district of each state of India.

\section{EXPERIMENTAL RESULTS}

In this work, we have evaluated our proposed model on various metrics like accuracy, mean absolute error and mean square error. The values of performance metrics are illustrated in Table 2.

Table 2: Performance Evaluation Table Showing Values of Accuracy, MAE, MSE and Loss

\begin{tabular}{|l|c|}
\hline \multicolumn{1}{|c|}{ Performance Parameters } & Results \\
\hline Accuracy & $99.19 \%$ \\
\hline MAE & 0.0157 \\
\hline MSE & 0.0078 \\
\hline Loss & 0.391 \\
\hline
\end{tabular}


Accuracy is measured as the difference between the predicted value and true value.

$$
\text { Acc }=\frac{y_{-} \text {pred }-y_{-} \text {true }}{n}
$$

Mean Absolute Error (MAE) measures the average of the difference between the predicted value and true value.

$$
\mathrm{MAE}=\frac{1}{n} \sum_{x_{y}} \mid y_{-} \text {pred }-y_{-} \text {true } \mid
$$

Mean Square Error measures the average of square of difference between predicted value and true value.

$$
\text { MSE }=\frac{1}{n^{2}} \Sigma_{x y}\left|y \_p r e d-y \_t r u e\right|^{2}
$$

Loss is measured using Binary Cross Entropy. Binary Cross Entropy function is used for binary classification problems.

$$
\mathrm{L}\left(y_{-} \text {true ,y_pred }\right)=\frac{1}{n} \sum_{i=0}^{n}\left(y_{-} \text {true } * \log \left(y_{-} \text {pred }\right)+\left(1-y_{-} \text {true }\right) * \log \left(1-y_{-} \text {pred }\right)\right)
$$

Where $\mathrm{n}$ is total number of data, $\mathrm{x}$ is set of parameters (state, district, season), $y$ represents label (crop).y_pred represents predicted value andy_true represents actual value.

During the training period, dataset is divided into two subclasses, one for training and the other for validation. $75 \%$ of data is used for training and the remaining $25 \%$ is used for validation. Figure 4illustrates the comparison of train and val. Here, 'Train' defines the trained sample's accuracy whereas 'Val' defines the accuracy of validation subclass.

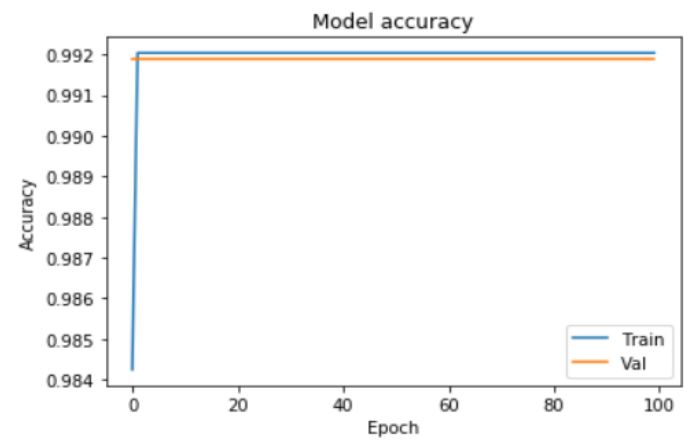

Figure.4: Train Versus Val Graph for Accuracy.

The comparison of loss during training and validation is illustrated in Figure 5.

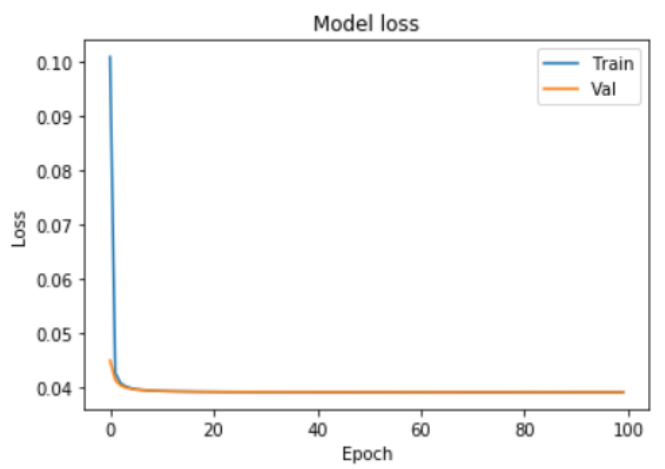

Figure 5: Train Versus Val Graph for Loss. 
We have compared our work with few previous works on the basis of accuracy obtained and the performance is illustrated in Table 3and Figure 6.

Table 3: Comparison of Our Work with Existing Work on the Basis of Accuracy

\begin{tabular}{|l|c|}
\hline \multicolumn{1}{|c|}{ Works } & Accuracy \\
\hline Our Work & $\mathbf{9 9 . 1 9 \%}$ \\
\hline Vivilya et al. [1] & $\mathbf{9 5 . 9 3 \%}$ \\
\hline Doshi et al. [11] & $\mathbf{9 1 \%}$ \\
\hline Pudumalar et al. [14] & $\mathbf{8 8 \%}$ \\
\hline Giritharan et al. [15] & $\mathbf{9 0 \%}$ \\
\hline
\end{tabular}

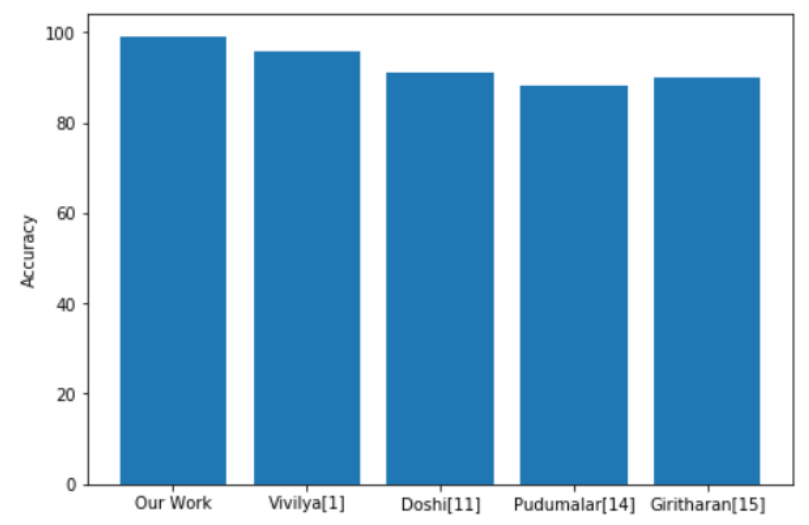

Figure 6: Comparison of Our Work with Existing Work on the Basis of Accuracy.

\section{CONCLUSIONS}

In this paper, we have designed a framework of crop prediction using Deep Neural Network. The proposed model predicts crops for different seasons and districts of each state on the basis of the production of crop from 1997 to 2015 . The model is trained using Deep Neural Network (DNN) having multiple layers. Sigmoid activation function and SGD optimizer are used in this model. The performance of the model is evaluated on various metrics like accuracy, mean absolute error, mean square error. The proposed model achieved accuracy of $99.19 \%$.

This work is mainly proposed to help farmers in choosing right crops according to the season and location so as to increase the production of crop. Our work achieved the accuracy of $99.19 \%$. In future, this work can be extended by taking more parameters like rainfall, temperature and soil type, etc.

\section{REFERENCES}

1. B. Viviliya, V. Vaidhehi, "The Design of Hybrid Crop Recommendation System using Machine Learning Algorithms," International Journal of Innovative Technology and Exploring Engineering (IJITEE) ISSN: 2278-3075, Volume-9 Issue-2, December 2019.

2. S. Khaki and L. Wang, “Crop Yield Prediction Using Deep Neural Networks," Front. Plant Sci. 10:621. doi: 10.3389/fpls.2019.00621, May 2019.

3. P.S. Maya Gopal, R. Bhargavi, “A novel approach for efficient crop yield prediction," Computers and Electronics in Agriculture, Volume 165, 2019, 104968, ISSN 0168-1699.

4. Mengistu, Abrham Debasu, and Dagnachew Melesew Alemayehu. "Robot for visual object tracking based on artificial neural network." International Journal of Robotics Research and Development (IJRRD) 6.1 (2016): 1-6. 
5. S. Chakrabarti, R. Braswell, N. Malizia, D. Sulla-Menashe, T. Cormier and M. Friedl, "In-Season Prediction of Crop Types In The us Great Plains Using Sequence Based Stochastic Models and Deep Learning," IGARSS 2019 - 2019 IEEE International Geoscience and Remote Sensing Symposium, Yokohama, Japan, 2019, pp. 5836-5839.

6. Chang, Wen-Yeau. "State of Charge Estimation for LFP Battery using Fuzzy Neural Network." International Journal of Electrical and Electronics Engineering Research (IJEEER) 6.5 (2016): 25-32.

7. S. M. Jajur, Soumya N. G., G. T. Raju, “Crop Recommendation using Machine Learning Techniques,” International Journal of Innovative Technology and Exploring Engineering (IJITEE) ISSN: 2278-3075, Volume-9 Issue-2S, December 2019.

8. K. Jha, A. Doshi, P. Patel, M. Shah, "A comprehensive review on automation in agriculture using artificial intelligence," Artificial Intelligence in Agriculture, Volume 2, 2019, Pages 1-12, ISSN 2589-7217.

9. S. Bhanumathi, M. Vineeth and N. Rohit, "Crop Yield Prediction and Efficient use of Fertilizers," 2019 International Conference on Communication and Signal Processing (ICCSP), Chennai, India, 2019, pp. 0769-0773.

10. N. H. Kulkarni, G. N. Srinivasan, B. M. Sagar and N. K. Cauvery, "Improving Crop Productivity Through A Crop Recommendation System Using Ensembling Technique," 2018 3rd International Conference on Computational Systems and Information Technology for Sustainable Solutions (CSITSS), Bengaluru, India, 2018, pp. 114-119.

11. Bagalkote, Ismail S., and Anup S. Vibhute."Multiresolution Analysis and Implementation of Grape Species Classification Using Neural Network." International Journal of Agricultural Science and Research 6.1 (2016): 37-46.

12. Tiwari, Preeti, Shukla, Piyush, "Crop Yield Prediction by Modified Convolutional Neural Network and Geographical Indexes, "International Journal of Computer Sciences and Engineering, 2018.

13. K. Vadnerkar, M. E. Patil, "Design and Implementation of Intelligent Recommendation System for Farmers using CNN and RNN," International Journal of Innovative Research in Science, Engineering and Technology, Vol. 7, Issue 11, November 2018.

14. Kumari, V. S. R., and P. Rajesh Kumar. "Cardiac arrhythmia prediction using improved multilayer perceptron neural network." Research and Develop.(IJECIERD) 3.4 (2013): 73-80.

15. Z. Doshi, S. Nadkarni, R. Agrawal and N. Shah, “Agro Consultant: Intelligent Crop Recommendation System Using Machine Learning Algorithms," 2018 Fourth International Conference on Computing Communication Control and Automation (ICCUBEA), Pune, India, 2018, pp. 1-6.

16. M. J. Mokarrama and M. S. Arefin, "RSF: A recommendation system for farmers," 2017 IEEE Region 10 Humanitarian Technology Conference (R10-HTC), Dhaka, 2017, pp. 843-850.

17. R. K. Rajak, A. Pawar, M. Pendke, P. Shinde, S. Rathod, A. Devare, “Crop Recommendation System to Maximize Crop Yield using Machine Learning Technique," International Research Journal of Engineering and Technology (IRJET), Volume: 04 Issue: 12 | Dec-2017.

18. S. Pudumalar, E. Ramanujam, R. H. Rajashree, C. Kavya, T. Kiruthika and J. Nisha, "Crop recommendation system for precision agriculture, " 2016 Eighth International Conference on Advanced Computing (ICoAC), Chennai, 2017, pp. 32-36.

19. G. Ravichandran and R. S. Koteeshwari, "Agricultural crop predictor and advisor using ANN for smartphones," 2016 International Conference on Emerging Trends in Engineering, Technology and Science (ICETETS), Pudukkottai, 2016, pp. 1-6.

20. G. Sanchez, Alberto, F. Soils, Juan, O. Bustamante, Waldo, "Predictive ability of machine learning methods for massive crop yield prediction," Spanish Journal of Agricultural Research, v. 12, n. 2, p. 313-328, April. 2014, ISSN 2171-9292.

21. www.data.gov.in 

